\title{
Chaix V., 2011 - Les églises romanes de Normandie, formes et fonctions
}

\section{Bernard Mandy}

\section{(2) OpenEdition}

\section{Journals}

Édition électronique

URL : http://journals.openedition.org/rao/3172

DOI : 10.4000/rao.3172

ISBN : 978-2-7535-5014-8

ISSN : $1775-3732$

\section{Éditeur}

Presses universitaires de Rennes

\section{Édition imprimée}

Date de publication : 31 décembre 2015

ISBN : 978-2-7535-5012-4

ISSN : 0767-709X

\section{Référence électronique}

Bernard Mandy, "Chaix V., 2011 - Les églises romanes de Normandie, formes et fonctions », Revue archéologique de l'Ouest [En ligne], 32 | 2015, mis en ligne le 28 avril 2016, consulté le 02 mars 2021. URL : http://journals.openedition.org/rao/3172 ; DOI : https://doi.org/10.4000/rao.3172 
- À partir de l'âge du Fer, l'archéologue se trouve confronté à des sources écrites, d'abord ténues et très indirectes mais progressivement de plus en plus prégnantes. Leur confrontation avec l'évidence archéologique a toujours été un exercice périlleux tant les logiques qui sous-tendent ces deux types d'informations sont différentes. Ici, on saura gré aux auteurs de ne pas avoir évacué le problème mais au contraire d'avoir su l'aborder avec lucidité et efficacité.

- Pour l'âge du Fer précisément, la révélation de ces dernières décennies en Centre-Bretagne aura incontestablement été la résidence aristocratique de Paule (découverte quasifortuitement en 1988), son évolution dans le temps et ses rôles dans la structuration d'un paysage très anthropisé ( $\mathrm{y}$ compris à travers une voirie déjà cohérente). Elle semble ainsi préfigurer le rôle qui reviendra plus tard à la ville galloromaine de Carhaix (laquelle, logiquement, occupe presque tout un chapitre entre l'agglomération proprement dite et ses prolongements que sont les aqueducs, la carrière de Locuon et le réseau routier).

- Parmi les spécificités de cet âge du Fer armoricain, on peut regretter que deux d'entre elles, les souterrains et les stèles, n'aient pas eu meilleure part dans l'ouvrage. Pour les premiers, un plan et une coupe paraissaient s'imposer à côté d'une photo malheureusement peu explicite. Quant aux secondes, elles n'apparaissent guère qu'à travers deux images ambigües alors que des monolithes spectaculaires (Sainte-Tréphine ou Plelauff) auraient permis, pour le grand public, d'illustrer (tout en l'exorcisant) le " mythe d'Obélix »!

- Le Moyen-Âge est l'occasion de présenter l'enceinte de Bressilien (elle aussi révélation de fouilles récentes) ou d'aborder avec lucidité la question toujours sensible des rap- ports ambigus entre monde breton et mondes franc puis carolingien et de survoler la mise en place de la féodalité à travers le semis des agglomérations et mottes castrales. Dommage cependant que l'ouvrage ne fasse qu'effleurer la question du Paléochrétien breton : outre la cloche de Paule évoquée, nous sommes ici aux confins du "triangle de Gildas » et, avec Sainte-Tréphine, en plein dans la saga de Comore (mais il est vrai que peu de découvertes sont venues alimenter la réflexion archéologique en ce domaine ces dernières décennies).

Au final, ce qui fera l'attractivité de cet ouvrage aux yeux de beaucoup, c'est aussi la qualité de sa présentation : maquette soignée, grande qualité d'une illustration abondante et variée (le crédit iconographique comporte plus de 40 noms). L'apport de la photo aérienne n'est plus à démontrer mais il se complète ici par quelques images Lidar (radar laser aéroporté), aussi spectaculaires que fourmillantes d'informations. Relevons également les très belles photographies (de sites et d'objets), d'assez nombreuses cartes (malheureusement pas toujours très lisibles et difficiles à caler par rapport à des fonds cartographiques actuels). Mais on retiendra surtout les nombreuses évocations de sites - en DAO ou sous forme de " vues d'artiste " - les unes et les autres très réussies.

En résumé, une fort belle production des éditions CoopBreizh qui intéressera un très large public - spécialisé ou non - désireux de s'informer sur le potentiel archéologique d'une région injustement méconnue ou d'obtenir une information de première main sur quelques sites qui font déjà référence chez les spécialistes.

Charles-Tanguy Le Roux

O'Sullivan Muiris (dir.), 2005 - Duma na nGiall - The Mound of the Hostages. Tara, Dublin, Wordwell/University College Dublin (School of Aechaeology), 308 p. (ISBN 1-8-6985-793-3).

Cette publication est emblématique à plus d'un titre. En raison du site concerné tout d'abord : à $40 \mathrm{~km}$ au nordouest de Dublin et à un peu plus de $20 \mathrm{~km}$ au sud-ouest de la célèbre boucle de la Boyne et de ses " monstres sacrés " du mégalithisme irlandais que sont New-Grange, Knowth et Dowth, la colline de Tara, du haut de ses $155 \mathrm{~m}$ d'altitude, commande un extraordinaire panorama sur la grande plaine centrale irlandaise. C'est, on le sait, un « haut-lieu " de l'histoire mythifiée de ce pays (d'où certaines initiatives "archéologiques ", parfois à la limite du farfelu dont le site fut l'objet comme rappelé dans le chap. I, (mais elles épargnèrent fort heureusement le "Mont des Otages " qui nous occupe ici). Les premières fouilles dignes de ce nom y furent dirigées en 1955-1956 par Sean P. O’Riordain. Cette entreprise pionnière à l'époque devait (un peu comme les fouilles de l'île Carn entreprises en Bretagne par P.-R. Giot à la même époque) déboucher de manière inattendue sur la découverte d'une tombe mégalithique enfouie sous un tumulus relativement " neutre " même s'il s'imposait dans le paysage avec ses $30 \mathrm{~m}$ de diamètre et $3,5 \mathrm{~m}$ de haut. Hélas interrompues par le décès du fouilleur, ces recherches devaient reprendre en 1959 sous l'impulsion de R. de Valera. Malgré plusieurs initiatives, matériel et documentation restèrent globalement sous-exploitées jusqu'au lancement 
d'un Discovery Programme Research on Tara en 1991 dont l'aboutissement fut la première édition de ce volume grâce à la ténacité de M. O'Sullivan qui s'était attaqué à ce fonds dès 1980

C'est d'ailleurs là que réside la seconde exemplarité de cette publication. Quiconque en a tenté l'expérience sait combien il peut être délicat de faire parler de la documentation ancienne, même recueillie dans toutes les règles de l'art de l'époque comme ce fut heureusement le cas à Tara : la problématique a changé, l'état des connaissances a évolué, les méthodes de travail et les moyens techniques aussi. En outre, certains protagonistes ayant disparu, leur pensée n'est plus accessible que partiellement et sous la forme figée de leurs derniers écrits.

On doit donc féliciter M. O'Sullivan et toute l'équipe constituée autour de lui (M. Herity, R. Cavanagh, U. Mattenberger, B. Coakley, M. Murray) pour avoir su faire revivre cette précieuse documentation. Comme relevé en tête du chapitre "Synthèse et conclusions " (p. 221), ce sont les données de terrain qui restaient les plus facilement exploitables (même si plans et coupes fleurent bon une "méthode Wheeler» de haute époque); par contre, les études post-fouille étaient devenues pour beaucoup relativement obsolètes. La place manque ici pour analyser en détails les tenants et aboutissants de cette remarque, mais c'est là une observation qui n'est pas isolée et dont la portée épistémologique mérite d'être méditée par tout responsable d'opération soucieux du devenir de son travail.

On se gardera bien de tenter ici un résumé global de l'ouvrage (il est d'ailleurs fort bien donné en prologue, p. vI); relevons simplement quelques points qui ouvrent des réflexions générales.

La continuité d'occupation d'un tel site, même si sa " phase mégalithique » est fort simple à côté de bien d'autres, n'a plus rien qui nous surprenne aujourd'hui. Après une faible trace de fréquentation mésolithique, les " choses sérieuses " y commencent dès le Early Neolithic irlandais (soit dans le $2^{\mathrm{e}}$ quart du IV millénaire B.C. cal.) par des structures en creux antérieures à la construction du monument. Comme en témoignent les 58 dates $14 \mathrm{C}$ fournies par le laboratoire de Groningen (dont 6 à l'accélérateur de particules), la fin de cette fréquentation recoupe l'utilisation primaire de la tombe mégalithique (3300-2900 B.C.) tandis qu'un hiatus de plus de trois siècles semble séparer celle-ci des fréquentations ultérieures : palissades et fosses, puis au moins 19 sépultures avérées du Early Bronze Age, incrus- tées dans le tumulus ou implantées à son pied même. L'une d'entre elles (le $\left.n^{\circ} 16\right)$ en est cependant distante de plus de $7 \mathrm{~m}$, tandis qu'une palissade s'échappe de la zone de fouilles. Comme souligné dans l'ouvrage, ceci laisse ouverte la possibilité d'autres structures non détectées au delà des quelque $700 \mathrm{~m}^{2}$ étudiés. C'est là une autre leçon : à l'heure où les contraintes de l'archéologie préventive restreignent parfois drastiquement les emprises étudiées, gardons à l'esprit que l'on ne peut bien comprendre de grandes structures (monumentales ou non) que dans un cadre élargi : impact paysager et/ou installations dans l'orbite de «structures-pilotes »... Et combien de sites anciennement fouillés ne seraient-ils pas à revoir dans cette optique?

Bien que profondément remodelé aux temps glaciaires, le substrat géologique de cette partie de l'Irlande est calcaire; en découle une excellente conservation des vestiges osseux (artefacts comme restes squelettiques - fauniques et surtout humains). Rien que pour la tombe centrale, près de 240 individus ont été reconnus; une telle situation laisse toujours le préhistorien habitué à la portion congrue du Massif armoricain admiratif devant la masse d'information ainsi disponible - et nostalgique face à celle dont il ne disposera jamais dans sa région.

$\mathrm{La}$ " haute significativité » du Mont des Hotages semble s'arrêter vers le milieu du II e millénaire B.C.; après, le relais est peut-être pris par les autres structures qui couronnent la colline de Tara, puis par la légende, avant l'oubli et le revival du XIX ${ }^{e}$ siècle.

Matériellement, cette publication se présente comme un très beau volume au format de 22 x $28 \mathrm{~cm}$. La typographie et la maquette sont soignées, l'illustration abondante et de qualité. Les plans issus de la documentation initiale ont été retavaillés avec efficacité par. U. Mattenberger (à qui l'on doit également l'essentiel des dessins d'objets). Les photos d'époque -noir et couleurs- sont, selon une tradition toute britannique, d'une remarquable qualité qui ne dépare pas à côté des photographies d'objets plus récentes. Un catalogue des trouvailles est fourni structure par structure (avec une numérotation un peu complexe, mais exploitation des fonds anciens oblige) et l'ouvrage se prolonge par dix " appendices » (dont le « 5 » rassemble les rapports spécialisés réunis à l'issue des recherches des années 1950). En résumé, un travail exemplaire dont l'intérêt dépasse très largement le microcosme des néolithiciens irlandais.

Charles-Tanguy Le Roux 